\title{
Construcción de una escala para determinar la utilidad de los Blogs en la educación superior
}

\author{
Verónica MARÍN-DÍAZ, Begoña E. SAMPEDRO-REQUENA y Esther VEGA-GEA
}

Universidad de Córdoba, España

(Recibido 15 Enero, 2016; Aceptado 13 Mayo, 2016)

RESUMEN: La incorporación de las herramientas digitales a la educación superior es incuestionable. En el caso de los blogs, estos son empleados de manera más latente, dada su facilidad de creación y empleo, y la no necesidad de poseer conocimientos informáticos para su creación. No obstante, nos cuestionamos si realmente son útiles para el desarrollo del currículo de los actuales estudios universitarios. Para determinar esto se ha procedido a construir una escala de valoración de la utilidad de los blogs en la educación superior. Para poder emplear la misma se ha procedido a realizar un estudio de la validez de la misma a través de la administración del mismo a una muestra de maestros de Educación Primaria en formación de la Universidad de Córdoba (N=162). El principal resultado alcanzado referente a la validez del instrumento señala que el análisis factorial exploratorio realizado a través de matrices policóricas a nivel de ítem refleja tres factores que miden las tres dimensiones abordadas. La principal conclusión a la que se ha podido llegar es que el conocimiento en la elaboración de los blogs se relaciona estadísticamente con el desarrollo del currículo, el desarrollo comunicativo presente en cualquier acto de aprendizaje, y el desarrollo informacional digital.

Palabras clave: Blog, educación superior, escala.

Construction of a scale to determinate the usefulness of Blogs in higher education

ABSTRACT: Nowadays, the inclusion of digital tools in higher education is unquestionable. For instance, blogs are used in a more latent way, given their ease of creation and use, and because no specific computer knowledge is needed. However, we question whether they are really useful for curriculum development of current University studies. To determine this, we proceeded to build a scale for assessing the usefulness of blogs in higher education. To use it, a study of its validity has carried out previously. In this study, the scale was handed out to a sample of Elementary School teachers in training, from the University of Cordoba $(N=162)$. Exploratory Factor Analysis were done over the retrieved data through polychoric matrices at item level, and the main result achieved indicated three factors that measure the three dimensions approached. The main conclusion that has been reached is that knowledge about blogs development is statistically related to the curriculum development, the communicative development present in any act of learning, and the digital informational development. Keywords: Blog; higher education; scale.

${ }^{1}$ Correspondencia: Universidad de Córdoba Facultad de Ciencias de la Educación Avda. San Alberto Magno s/n. 14004-Córdoba (Spain). E-mail: vmarin@uco.es. 
De acuerdo con Huang, Hood y Yoo (2013) la Web 2.0 no es una tecnología, es una forma de entender el cambio social que hoy se vive, por lo que la actitud hacia ella será vital en la relación que se construya con o hacia la misma. Prueba de ello ha sido su rápido progreso, lo que la ha convertido en piedra angular del avance de la sociedad.

En el ámbito educativo, poco a poco han ido haciéndose hueco, tratando de demostrar que el aprendizaje puede llegar a ser más efectivo con las TIC siempre y cuando, esa actitud de la que hablamos, sea receptiva y abierta. Una de las herramientas que más incidencia está teniendo en la educación, a cualquier nivel, son los blogs (Deng y Yuen, 2012), los cuales se han ido haciendo un hueco en los procesos educativos de forma llamativa, prueba de ello son las investigaciones que manifiestan su presencia (Hevia y García, 2006; Chong, 2010; Mansor, 2011, 2012; Marín, 2013; Osman y Koh, 2013;Marín, Muñoz y Sampedro, 2014; Marín y Gómez, 2015; Marín y Sampedro, 2016; Quintanal, 2011), reflejando que son proveedores de aprendizajes de carácter más flexible, abierto, interactivo y dinámico.

Autores como Noel (2015) afirman que los blogs en sí, no ayudan a crear lazos de conocimiento entre los conceptos, sentencia esta que se ve apoyada por los datos alcanzados por Brown en 2012, los cuales revelan que algunos docentes no perciben que las tecnologías de la información y la comunicación, ni las herramientas Web 2.0 sean apropiadas para la enseñanza en todo momento o contexto. Esta realidad pone de manifiesto que los blogs, pueden ser elementos que no potencien el desarrollo del currículo. En este sentido González y García (2011) al igual que Lai y Chen (2011) indican que el mantenimiento de estos implica que el docente ha de emplear un elevado número de horas para que este realmente cumpla su objetivo. En esta línea podemos decir que los blogs, en general, no propician las habilidades de escritura y lectura, se desatiende la privacidad de los post, los cuales están sujetos a la voluntariedad del gestor del mismo, para ser bloqueados o eliminados, el docente pierde la propiedad de su conocimiento (Zeng y Harris, 2005; Lain y Chen, 2011). El estudio llevado a cabo por Tapia y Escudero (2014) con estudiantes universitarios, reflejó que estos preferían la comunicación personal cara a cara, frente a la indirecta que ofrecen los blogs, así la participación en los debates que se tratan de provocar en los blogs tienen una escasa participación por parte de los alumnos (Divitini, Hangalokken y Morken, 2005)

No obstante, también encontramos visiones menos apocalípticas de esta herramienta, como es la aportada por Goktas y Demirel (2012), quienes indican que los blogs otorgan de ilimitadas oportunidades para experimentar, pues otorgan una nueva perspectiva a las herramientas Web 2.0. Estos pueden ser vistos como facilitadores de oportunidades de comunicación, construcción de pensamientos críticos, abstractos y analíticos y conocimientos profundos (Du y Wagner, 2006; Akçay y Arslan, 2010;Hall y Davinson, 2007;Mansor, 2011), la auto-reflexión (Laru, Naykki y Järrela, 2012), permite incorporar temas de actualidad, como un recurso o material virtual al servicio del docente (Fernández, Bonatto y Garrido, 2013), promueven la creatividad (Marín y Gómez, 2015), entre otros aspectos.

Por todas estas circunstancias, consideramos al igual que Osman y Koh (2013) que el estudio de la validez de los blogs como herramienta para desarrollar el currículo se encuentra en sus inicios, de ahí la necesidad de construir un instrumento que nos ayude a determinar este aspecto. Desde esta perspectiva y tras realizar una revisión literaria en torno a los blogs se 
procedió a la construcción de un cuestionario y a validar el mismo, con el fin de poder establecer como herramienta curricular en las aulas universitarias los blog.

\section{Método}

El presente estudio se fundamenta en un paradigma positivista, considerando las tres corrientes que existen en investigación educativa (Meza, 2002; Sabariego, 2012); en concreto, desde una perspectiva metodológica, se caracteriza por un lado, en medir los cambios que se producen en las variables independientes correspondiendo a un diseño ex post facto y descriptivo (Mateo, 2012); y, por otro, en un diseño instrumental al intentar explicar las relaciones entre las variables latentes de respuesta que subyacen en los datos extraídos (Domínguez Lara, 2014).

Con el presente estudio se persiguen varios objetivos. El primero de ellos fue validar una escala que mida si el uso de los blogs sirve para desarrollar el currículo o no. El segundo objetivo pretendía analizar la diferencia de medias entre las diferentes dimensiones del instrumento "utilidad del uso de los blog en educación” y las variables independientes: sexo, curso, uso de los dispositivos móviles utilizados y conocimiento de elaboración de blogs.

\section{Instrumento}

La construcción de la escala se realizó en un proceso de dos fases. La primera de ellas consistió en la elaboración de un listado inicial de ítems, cuya finalidad era elaborar un conjunto de ítems relacionados con la utilidad de los blogs para desarrollar el currículo. Para ello se obtuvo información principalmente de dos fuentes: a) observación y conversación con el alumnado del Grado de Educación Primaria y b) consulta de instrumentos de medida relacionados con la utilidad de los blogs. Este proceso dio como resultado una escala inicial compuesta de 22 ítems.

La segunda fase fue la consulta al grupo de expertos, con ella se pretendía conocer la valoración de los diferentes ítems de la escala por diferentes expertos. Para esta evaluación de los ítems se contactó con varios expertos en el ámbito de la tecnología educativa. Concretamente, participaron 14 expertos. 4 de la Universidad de Sevilla (España), 2 de la Universidad de Veracruz (México), 5 de la Universidad de Córdoba (España), y 3 de la Universidad de Concepción (Chile).

El instrumento para valorar los ítems de la escala se elaboró ad hoc, y se les envió a los expertos vía email. El mismo se centraba en valorar la relevancia y claridad de los ítems. Estos aspectos se medían con una escala de 1 a 5 , donde 1 equivalía a nada relevante y nada claro y 5 a muy relevante y muy claro.

Una vez evaluados los ítems por los expertos, y con el fin de interpretar las respuestas dadas por los mismos, referentes a las variables de relevancia de los ítems en la escala y claridad -referido a la facilidad para comprender lo que se pregunta-, se eligió como corte de selección de los ítems aquellos que presentaran una media de respuesta a partir de 4, tanto en la variable claridad como en la de relevancia. Se eligió este valor como punto medio de la escala, en lugar del 2.5 para darle una mayor rigurosidad a la selección de los ítems (Moreno, 2015). Tras el análisis de las respuestas dadas por los expertos, la escala inicial se mantuvo, ya que las 
puntuaciones de todos ellos alcanzaron una puntuación de 4, tanto en claridad como en relevancia. Por tanto, la escala definitiva quedó compuesta por un total de 22 ítems, y se la denominó Utilidad de los blogs en Educación (UBE) medidos en escala Likert de 5 puntos (1 totalmente inútiles a 5 totalmente útiles). La consistencia interna del instrumento fue $\alpha=.94$.

\section{Muestra}

La muestra estuvo compuesta por 162 estudiantes de $1^{\circ}$ y $2^{\circ}$ de Grado de Educación Primaria seleccionados a través de un muestreo intencional en la Facultad de Ciencias de la Educación de la Universidad de Córdoba (España). El rango de edad estuvo comprendido entre 18 y 44 años $(M .=20.16$, D.E.= 3.26), siendo un $66 \%$ mujeres, $n=107)$. Un $59.3 \%$ de los estudiantes cursaban segundo y un $40.7 \%$ primero. El centro educativo de procedencia de los participantes era mayoritariamente público (67.9\%).

Atendiendo al dispositivo de conexión difería, en función del uso al que iba destinado, el $66 \%$ indicó emplear el teléfono móvil, sin embargo, cuando el uso era destinado al ámbito educativo, los estudiantes utilizaban más el ordenador portátil (87 \%). Un 48.1\% del alumnado tenía conocimiento sobre la elaboración de los blogs, mientras que un 8.6\% su desconocimiento era total al respecto.

\section{Procedimiento}

El instrumento fue administrado en sesiones ordinarias de clase, y la duración media de finalización del cuestionario fue de 15 minutos. Los investigadores del estudio fueron los encargados de recoger los datos en la Universidad. Se contó con la colaboración de los profesores de la Titulación del Grado de Educación Primaria para entrar en sus clases a recoger los datos.

\section{Análisis de datos}

Los análisis estadísticos del estudio se realizaron en dos fases. En primer lugar, para comprobar la evidencia de la dimensionalidad del instrumento de utilidad de los blogs en el desarrollo del currículo, y seleccionar los ítems definitivos que compondrían el instrumento definitivo, se realizó un Análisis Factorial Exploratorio (AFE), utilizando para ello el software estadístico Factor 10.3 (Lorenzo-Seva y Ferrando, 2006). Se aplicó una extracción basada en el método de estimación Unweighted Least Squares (ULS), el método de rotación Promax, tomando como base la matriz de correlaciones policóricas, ya que ésta es recomendada para muestras con una distribución no normal (Muthen y Kaplan, 1992) y cuando la escala de medida de los ítems es de naturaleza ordinal (Bryant y Satorra, 2012). Para determinar el número de factores se aplicó el Hull Method (Lorenzo-Seva, Timmerman y Kiers, 2011).

En segundo lugar, se realizaron análisis descriptivos de las dimensiones de la escala, así como análisis de varianza para comprobar si existía relación entre las dimensiones de la escala y las variables independientes: sexo, curso, dispositivos de conexión utilizados y conocimiento de elaboración de los blogs. Para ello se llevaron a cabo pruebas no paramétricas U de Mann- 
Whitney (paramétrica t-student) y $H$ de Kruskal Wallis (paramétrica ANOVA), utilizando el paquete estadístico SPSS 20.

\section{Resultados}

\section{Pruebas de normalidad de la muestra.}

El tamaño muestral fue el adecuado ya que siguiendo a Nunnally (1978), es recomendable que la muestra sea al menos, cinco veces mayor que el número de ítems. En el presente estudio, el instrumento se compuso de 22 ítems y la muestra total (sin datos perdidos) fue de 162 participantes, por lo que se podría considerar que el tamaño muestral fue suficiente.

En cuanto a la distribución de la muestra, los índices de asimetría y curtosis indicaron frecuencias de respuestas que seguían una distribución no normal ya que los valores absolutos de algunos de los ítems están por encima de 1 (Muthen y Kaplan, 1992). Para una mayor seguridad, puesto que muchos de los ítems puntuaron por debajo de 1 tanto en la curtosis como en la asimetría, se comprobó el índice multivariado de curtosis o coeficiente de Mardia (Mardia, 1970). Este alcanzó un valor de 580.120, resultado muy superior a lo recomendado para considerar la condición de normalidad multivariante $(\leq 70)$. Por todo ello, se podría afirmar que las variables analizadas violaban el supuesto de normalidad multivariante (ver tabla 1).

Tabla 1. Pruebas de normalidad de los ítems del instrumento

\begin{tabular}{llcccc}
\multicolumn{1}{c}{ ITEMS } & $\mathrm{M}$ & $\mathrm{D} . \mathrm{T}$. & Asimetría & Curtosis* \\
\hline 1. Valor que le otorgo a los blogs en Educación & 4.17 & .71 & -.77 & 1.48 \\
2. Permiten seguir mejor la asignatura & 4.18 & .68 & -.48 & .06 \\
3. Ayudan a resolver mejor dudas & 4.06 & .72 & -.38 & -.16 \\
4. & Estimulan la búsqueda de información & 4.22 & .76 & -.64 & -.21 \\
5. Ayudan a crear una visión crítica sobre la información & 3.80 & .81 & -.03 & -.78 \\
6. Ayudan a expresar ideas y opiniones & 4.41 & .71 & -1.09 & .96 \\
7. Fomentan la participación del alumnado en la asignatura & 4.26 & .83 & -1.18 & 1.69 \\
8. Fomentan el pensamiento creativo & 4.35 & .76 & -1.03 & .052 \\
9. Ayudan a desarrollar la competencia informacional & 4.28 & .73 & -.58 & -.59 \\
10. Ayudan a desarrollar la competencia digital & 4.62 & .59 & -1.28 & .62 \\
11. Potencian el trabajo de grupo cooperativo y colaborativo & 4.09 & .88 & -.60 & -.55 \\
12. Ayudan a tener una visión positiva del proceso de enseñanza & 4.05 & .69 & -.29 & -.19 \\
13. Ayudan a tener una visión positiva del proceso de aprendizaje & 4.10 & .70 & -.35 & -.25 \\
14. Ayudan a vincular los contenidos curriculares con la realidad de la sociedad & 3.93 & .79 & -.47 & .31 \\
15. Ayudan a ampliar los contenidos desarrollados en el aula & 4.40 & .66 & -.79 & .09 \\
16. Favorecen trabajar de forma transversal el currículo & 4.03 & .81 & -.68 & .51 \\
17. Potencian la comprensión lectora & 4.09 & .82 & -.63 & -.13 \\
18. Potencian la comprensión escrita & 4.00 & .89 & -.62 & -.13 \\
19. Potencian las habilidades de comunicación digital & 4.01 & .84 & -.44 & -.53 \\
20. Ayudan a romper con las barreras culturales, sociales o económicas & 3.74 & .80 & -.08 & -.54 \\
21. Potencian el sentido de comunidad o grupo clase & 3.94 & .82 & -.28 & -.64 \\
22. Potencian los procesos de reflexión en los estudiantes & 4.14 & .74 & -.33 & -.85 \\
& & $\mathrm{~N}=162$ & $\mathrm{C} . \mathrm{M} .=588.120$ & $\mathrm{p}=0.0000 * *$ \\
\hline
\end{tabular}

*Centrado en el cero (zero centered) 


\section{Matriz de correlaciones de los ítems de la escala}

Se pidió la matriz de correlaciones policóricas en lugar de la matriz de correlaciones de Pearson, ya que cuando la distribución de la muestra no es normal es más recomendado examinar las correlaciones entre los ítems de la escala mediante la matriz de covarianza o correlaciones policóricas (Tabla 2).

Como se puede observar en la tabla 2, la mayoría de las correlaciones presentaron valores significativos (por encima .30) y positivos, lo que apuntaba la existencia de relación entre los diferentes ítems que conformaban la escala.

Tabla 2. Matriz de correlaciones policóricas

\begin{tabular}{|c|c|c|c|c|c|c|c|c|c|c|c|c|c|c|c|c|c|c|c|c|c|c|}
\hline & 1 & 2 & 3 & 4 & 5 & 6 & 7 & 8 & 9 & 10 & 11 & 12 & 13 & 14 & 15 & 16 & 17 & 18 & 19 & 20 & 21 & 22 \\
\hline 1 & 1 & & & & & & & & & & & & & & & & & & & & & \\
\hline 2 & .538 & 1 & & & & & & & & & & & & & & & & & & & & \\
\hline 3 & .438 & .609 & 1 & & & & & & & & & & & & & & & & & & & \\
\hline 4 & .395 & .448 & .477 & 1 & & & & & & & & & & & & & & & & & & \\
\hline 5 & .312 & .451 & .423 & .484 & 1 & & & & & & & & & & & & & & & & & \\
\hline 6 & .359 & .300 & .209 & .304 & .334 & 1 & & & & & & & & & & & & & & & & \\
\hline 7 & .500 & .487 & .386 & .423 & .342 & .487 & 1 & & & & & & & & & & & & & & & \\
\hline 8 & .353 & .310 & .350 & .579 & .350 & .481 & .487 & 1 & & & & & & & & & & & & & & \\
\hline 9 & .323 & .389 & .329 & .2 .50 & .287 & .363 & .394 & .435 & 1 & & & & & & & & & & & & & \\
\hline 10 & .453 & .400 & .372 & .443 & .323 & .476 & .505 & .599 & .517 & 1 & & & & & & & & & & & & \\
\hline 11 & .329 & .430 & .408 & .341 & .257 & .251 & .337 & .359 & .271 & .407 & 1 & & & & & & & & & & & \\
\hline 12 & .398 & .542 & .481 & .469 & .430 & .316 & .468 & .417 & .451 & .414 & .448 & 1 & & & & & & & & & & \\
\hline 13 & .477 & .611 & .533 & .550 & .458 & .358 & .463 & .458 & .423 & .431 & .400 & .754 & 1 & & & & & & & & & \\
\hline 14 & .439 & .524 & .479 & .478 & .321 & .350 & .358 & .379 & .346 & .513 & .428 & .597 & .621 & 1 & & & & & & & & \\
\hline 15 & .513 & .579 & .566 & .449 & .365 & .437 & .495 & .407 & .377 & .548 & .427 & .513 & .528 & .521 & 1 & & & & & & & \\
\hline 16 & .382 & .543 & .528 & .489 & .430 & .440 & .510 & .448 & .318 & .489 & .283 & .531 & .537 & .541 & .580 & 1 & & & & & & \\
\hline 17 & .375 & .380 & .361 & .445 & .403 & .417 & .263 & .426 & .314 & .530 & .348 & .413 & .437 & .384 & .405 & .426 & 1 & & & & & \\
\hline 18 & .408 & .283 & .326 & .360 & .274 & .381 & .282 & .413 & .313 & .460 & .403 & .436 & .453 & .359 & .339 & .289 & .703 & 1 & & & & \\
\hline 19 & .365 & 3.57 & .370 & .369 & .301 & .252 & .389 & .434 & .317 & .553 & .380 & .456 & .459 & .464 & .383 & .369 & .434 & .498 & 1 & & & \\
\hline 20 & .313 & .457 & .401 & .325 & .435 & .312 & .334 & .188 & .291 & .289 & .325 & .389 & .485 & .362 & .401 & .494 & .347 & .236 & .305 & 1 & & \\
\hline 21 & .355 & .525 & .474 & .382 & .392 & .281 & .456 & .359 & .319 & .426 & .571 & .490 & .454 & .394 & .512 & .380 & .414 & .415 & .408 & .463 & 1 & \\
\hline 22 & .386 & .469 & .423 & .473 & .511 & .370 & .490 & .413 & .360 & .409 & .396 & .560 & .493 & .453 & .514 & .460 & .436 & .413 & .514 & .421 & .552 & 1 \\
\hline
\end{tabular}

\section{Análisis Factorial Exploratorio (AFE)}

Se realizó un análisis factorial exploratorio (AFE) basado en el método de extracción Unweighted Least Squares (ULS) con rotación promax, utilizando el método Kaiser-MeyerOlkin(KMO) y el método heurístico, Cattell'sScree Test. La medida de adecuación muestralKaiser-Meyer-Olkin (KMO), con un valor de .89 y el test de esfericidad de Bartlett's, $\mathrm{X}^{2}(45)=1613.48, \mathrm{p}<.000$ estadísticamente significativo, corroboraron la pertinencia de realizar un AFE. En el análisis se solicitaron tres factores, uno para cada una de las dimensiones 
medidas, los cuales explicaban una varianza total del 56.03\%. Asimismo, se probó el análisis con otros números de factores, pero los resultados obtenidos no fueron satisfactorios, por lo que se optó por la solución obtenida de 3 factores. Todos los ítems revelaron comunalidades por encima de .33, y cargas factoriales mayores a .40, a excepción del ítem 22 "Potencian los procesos de reflexión en los estudiantes", cuyo peso factorial fue de .34. La tabla 4 muestra los factores e ítems de la escala, describiendo en la misma, los índices de fiabilidad tanto de la escala global como de las diferentes dimensiones, las comunalidades y pesos factoriales, y el porcentaje de varianza explicada por cada uno de los factores que componen la escala.

Tabla 3. Análisis factorial exploratorio sobre la utilidad del uso de los blogs

\begin{tabular}{|c|c|c|c|}
\hline Factores & Items & Comunalidad & $\mathrm{R}^{2}$ \\
\hline Desarrollo Currículo $(\alpha=.92)$ & 1. Valor que le otorgo a los blogs en Educación & .38 & .40 \\
\hline \multirow[t]{13}{*}{ 44.94\% varianza explicada } & 2. Permiten seguir mejor la asignatura & .65 & .92 \\
\hline & 3. Ayudan a resolver mejor dudas & .52 & .79 \\
\hline & 4. Estimulan la búsqueda de información & .43 & .40 \\
\hline & 5. Ayudan a crear una visión crítica sobre la información & .34 & .52 \\
\hline & 11. Potencian el trabajo de grupo cooperativo y colaborativo & .35 & .44 \\
\hline & $\begin{array}{l}\text { 12. Ayudan a tener una visión positiva del proceso de } \\
\text { enseñanza }\end{array}$ & .58 & .68 \\
\hline & $\begin{array}{l}\text { 13. Ayudan a tener una visión positiva del proceso de } \\
\text { aprendizaje }\end{array}$ & .63 & .73 \\
\hline & $\begin{array}{l}\text { 14. Ayudan a vincular los contenidos curriculares con la } \\
\text { realidad de la sociedad }\end{array}$ & .48 & .56 \\
\hline & 15. Ayudan a ampliar los contenidos desarrollados en el aula & .56 & .57 \\
\hline & 16. Favorecen trabajar de forma transversal el currículo & .54 & .55 \\
\hline & $\begin{array}{l}\text { 20. Ayudan a romper con las barreras culturales, sociales o } \\
\text { económicas }\end{array}$ & .36 & .67 \\
\hline & 21. Potencian el sentido de comunidad o grupo clase & .47 & .60 \\
\hline & 22. Potencian los procesos de reflexión en los estudiantes & .49 & .51 \\
\hline \multirow{2}{*}{$\begin{array}{l}\text { Desarrollo Comunicativo } \\
\qquad(\alpha=.83)\end{array}$} & 6. Ayudan a expresar ideas y opiniones & .42 & .68 \\
\hline & 7. Fomentan la participación del alumnado en la asignatura & .52 & .59 \\
\hline \multirow[t]{3}{*}{$6.42 \%$ varianza explicada } & 8. Fomentan el pensamiento creativo & .58 & .76 \\
\hline & 9. Ayudan a desarrollar la competencia informacional & .32 & .42 \\
\hline & 10. Ayudan a desarrollar la competencia digital & .65 & .72 \\
\hline \multirow{2}{*}{$\begin{array}{l}\text { Desarrollo Informacional Digital } \\
\qquad(\alpha=.86)\end{array}$} & 17. Potencian la comprensión lectora & .58 & .65 \\
\hline & 18. Potencian la comprensión escrita & .79 & .95 \\
\hline \multirow[t]{2}{*}{ 5.18\% varianza explicada } & 19. Potencian las habilidades de comunicación digital & .42 & .38 \\
\hline & Alpha de Cronbach estandarizado de la escala glob & $=0.94$ & \\
\hline
\end{tabular}

El primer factor, se denominó Desarrollo Currículo $(\alpha=.92)$. Este factor explö́ un 44.94\% de la varianza total, e incluía un total de catorce ítems, que describían la utilidad de los blogs en tareas o actividades académicas o relacionadas con la educación. El segundo factor, denominado Desarrollo Comunicativo $(\alpha=.83$ ), explicó un $6.42 \%$ de la varianza total y estaba compuesto por un total de cinco ítems relacionados con el desarrollo de la expresión y comunicación. Finalmente, el tercer factor denominado Desarrollo Informacional Digital $(\alpha=$ .86), explicó un 5.18\% de la varianza total, configurado por tres ítems (Tabla 3). 
En la tabla 4 se muestra la matriz de correlaciones interfactores. Todos los componentes presentaron correlaciones por encima de .30. La mayor correlación entre los factores de la escala se encontró entre el factor 1 y el factor 2 (.724).

Tabla 4. Matriz de correlaciones inter-factores

\begin{tabular}{lccc} 
& Factor 1 & Factor 2 & Factor 3 \\
\hline Factor 1 & 1 & & \\
Factor 2 & .724 & 1 & \\
Factor3 & .593 & .612 & 1 \\
\hline
\end{tabular}

Las correlaciones observadas en la tabla 4, apuntan que existe una alta relación entre todos los factores que componen la escala, pero en ningún caso superior a .80. Por tanto, los datos indicaron que aunque es evidente la relación entre los factores de la escala, estos no miden lo mismo.

Diferencias entre los factores de la escala y variables: sexo, curso, uso dispositivos móviles y conocimiento de elaboración de blogs.

En este apartado se presentan los resultados correspondientes al tercer objetivo propuesto en el estudio: comparar la percepción de utilidad del uso de los blog en educación en función de las variables: sexo, curso, uso de dispositivos móviles generales de conexión y para tareas académicas, y conocimiento de elaboración de los blogs.

Para ello se realizaron análisis de contraste de hipótesis para ver si las respuestas diferían en función de las variables de clasificación (citadas anteriormente). Puesto que no se cumplían los requisitos que exigen las pruebas paramétricas (normalidad de la distribución muestral) se han realizado pruebas no paramétricas. Concretamente, se llevaron a cabo estadísticos de contraste $U$ de Mann-Whitney para dos muestras independientes (sexo, curso), y estadísticos de contraste $H$ de Kruskal Wallis para más de dos muestras independientes.

- Diferencias en el factor 1: Desarrollo del currículo.

En el primer factor de la escala, únicamente se encontraron diferencias significativas con la variable conocimiento en la elaboración de los blogs. La prueba $H$ de Kruskal Wallis señaló diferencias significativas $\left[\chi^{2}(3, N=162)=20.207, p=.000\right]$. Concretamente, las diferencias se encontraron entre el grupo de los que dijeron que conocían totalmente la elaboración de los blogs y el resto de grupos (desconocimiento total, conocimiento y bastante conocimiento), los que tenían un conocimiento total de la elaboración de los blogs consideraban más útil el uso de los blogs para desarrollar el currículo que el resto de los grupos estudiados. No se encontraron diferencias significativas en relación a las variables: sexo $(p=.66)$; curso $(p=.23)$; dispositivo conexión general $(p=.79)$; y dispositivo conexión para realizar tareas $(p=.40)$.

- Diferencias en el factor Desarrollo Comunicativo.

En el segundo factor, se encontraron diferencias significativas en relación a la variable curso y la variable conocimiento sobre la elaboración de los blogs. Respecto a la variable curso, los datos de la prueba $U$ de Mann-Whitney indicaron diferencias significativas $(Z=-2.204, p$ $=.02$ ), lo que significa que el curso influye en la percepción de utilidad que se le asigna al uso de los blogs en la dimensión referente al Desarrollo Comunicativo, siendo que los estudiantes de $2^{\circ}$ del Grado de Educación Primaria atribuyeron una mayor utilidad al uso de los blogs que los estudiantes de $1^{\circ}$ de Primaria. 
Asimismo, la prueba $H$ de Kruskal Wallis apuntó diferencias significativas $\chi^{2}(3, N=$ $162)=15.223, p=.002$ ] entre el factor 2: Desarrollo Comunicativo y la variable conocimiento de elaboración de los blogs. El grupo de los que dijeron que conocían totalmente la elaboración de los blogs le asignaron una mayor utilidad al uso de los blogs en relación al desarrollo comunicativos que los que afirmaron que únicamente lo conocían.

No se encontraron diferencias significativas entre la variable Desarrollo Comunicativo y las variables sexo, curso, y conexión a dispositivos.

- Diferencias en el factor Desarrollo Informacional Digital.

No se encontraron diferencias significativas en ninguna de las variables estudiadas (sexo, curso, conexión y conocimiento de elaboración de los blogs) en relación a la variable Desarrollo Información Digital.

\section{Conclusiones}

Considerados los blogs por algunos autores (Dos y Demir, 2013) como una de las herramientas más populares de los social media, se ha procedido a contrastar este aspecto a través de la construcción de un cuestionario, que, como ya hemos indicado con anterioridad, determinará si realmente son una herramienta educativa que permite el desarrollo del currículo, comunicativo e informacional y digital de los estudiantes. La prueba de validez realizada a este, mediante un análisis factorial exploratorio basado en matrices policóricas (Domínguez Lara, 2014), pone de manifiesto la existencia de tres factores que miden las tres dimensiones abordadas en este estudio: desarrollo curricular; desarrollo comunicativo; y desarrollo informacional digital.

En este sentido, el análisis de las matrices policóricas, basado en supuestos de normalidad se puede realizar ya que está justificado para el caso de los estudios exploratorios, puesto que se ha demostrado que las soluciones obtenidas no difieren de las obtenidas en las correlaciones de Pearson ni de las correlaciones tetracóricas (Freiberg, Stover, De la Iglesia y Fernández, 2013), aspecto que se refleja en este estudio. Por tanto, el análisis factorial exploratorio realizado explica mediante las variables latentes el conjunto de variables advertidas, produciendo validez en el constructo del instrumento diseñado ad hoc.

Por otro lado, existe evidencia de relación entre el conocimiento de elaboración de los blogs y el desarrollo del currículo; el desarrollo comunicativo; y el desarrollo informacional digital.

La relación pedagógica entre el conocimiento, en este caso de elaboración de blogs y la comunicación en cualquier proceso de aprendizaje, es algo evidente (Rojas y Parra, 2009)dado que el acto dialógico producido entre los agentes de la transformación de la mera información en saber provocan la movilidad de diversos estilos cognitivos que propician este.

La correspondencia existente entre el desarrollo del currículo y el conocimiento en la elaboración de blogs se sustenta en el hecho de que cada vez es mayor su empleo en niveles primarios de la educación (Marín, Muñoz y Sampedro, 2014; Marín y Gómez, 2015).

Por último, la reciprocidad que surge entre el desarrollo informacional digital y el conocimiento en la elaboración de blog es similar al encontrado por Luik, Voltri, Taimalu y Kakl (2011) o Marín (2013). 


\section{Referencias}

Akçay, A., y Arslan, A. (2010). Theusing of blogs in Turkisheducation. Procedia Social and BehavioralSciences, 2, 1195-1199.

Brown, S. (2012). Seeing web 2.0 in context: a study of academic perceptions.The Internet and Higher Education, 15(1), 50-57.

Bryant, F. B., y Satorra, A. (2012). Principles and practice of scaled difference chi-square testing. Structural Equation Modeling: A Multidisciplinary Journal, 19(3), 372-398.

Chong, E. K. M. (2010). Using blogging to enhance the initiation of students into academic research. Computers \& Education, 55, 798-807.

Deng, L., y Yuen, A. H. K. (2012). Understanding student perceptions and motivation towards academic blogs: An exploratory study. Australasian Journal of Educational Technology, 28(1), 48-66.

Divitini, M., Haugalokken, O., y Morken, E. M. (2005). Blog to support learning in the field: lessons learned from a fiasco. In Proceedings of Fifth IEEEE International Conference on Advanced Learning Technologies (ICALAT'05).

Dominguez Lara, S.A. (2014). ¿Matrices Policóricas/Tetracóricas o Matrices Pearson? Un estudio metodológico. Revista Argentina de Ciencias del Comportamiento, 6(1), 39-48.

Dos, B., y Demir, S. (2013). TheAnalysis of the Blogs Created in a Blended Course through the Reflective Thinking Perspective. Educational Sciences: Theory \& Practice, 13(2), 13351344.

Du, H. S., y Wagner, C. (2006). Weblog success: exploring the role of technology. International Journal of Human Computer-Studies, 64 (9), 789-798.

Fernández, L.N., Bonatto, L., y Garrido, C. (2013).Capoeira e temas transversais: avaluaçao de un blog didáctico para las aulas de educaçao fisica. ETD, 15(1), 87-106

Freiberg, A., Stover, J.B., De la Iglesia, G., y Fernández, M. (2013). Correlaciones policóricas y tetracóricas en estudios factoriales exploratorios y confirmatorios. Ciencias Psicológicas VII(2), 151-164.

Goktas, Y., y Demirel, T. (2012). Blog-enhanced ICT courses: examining their effects on prospective teachers' ICT competencies and perceptions. Computers \&Education, 58, 908-917.

González, R., y García, F. (2011). Recursos eficaces para el aprendizaje en entornos virtuales en el Espacio Europeo de Educación Superior. ESE, Estudios de Educación, 20, 161-180

Hall, H., y Davison, B. (2007). Social software as support in hybrid learning environments: the value of the blog as a tool for reflective learning and peer support. Library and Information Science Research, 25(2), 163-187.

Hevia, S., y García, R. (2006).Proyecto WAU: Un caso práctico de diseño inclusivo. Revista No solo usabilidad, vol.5. Recuperado el 20 de marzo de 2013 desde http://www.nosolousabilidad.com/articulos/proyecto_wau.htm

Huang, W. H., Hood, D. W., y Yoo, S. J. (2013). Gender divide and acceptance of collaborative web 2.0 applications for learning in higher education. The Internet and Higher Education,16, 57-65.

Lai, H.-M., y Chen, C.P. (2011).Factors influencing secondary schools teachers' adoption of teaching blogs. Computers \& Education, 56, 948-960. 
Laru, J., Näykki, P., y Järvelä, S. (2012). Supporting small-group learning using multiple web 2.0 tools: a case study in the higher education context. The Internet and Higher Education 15, 29-38.

Lorenzo-Seva, U., y Ferrando, P.J. (2006). FACTOR: A computer program to fit the exploratory factor analysis model. Behavior Research Methods Instruments \& Computers,38(1), 88-91

Lorenzo-Seva, U., Timmerman, M. E., y Kiers, H. A. (2011). The Hull method for selecting the number of common factors. Multivariate Behavioral Research, 46(2), 340-364

Luik, P., Voltri, O., Taimalu, M., y Kakl, K. (2011).On the use of student teacher blogs during teaching practice. Procedia Social and Behavioral Sciences, 11, 165-169.

Mansor, A. Z. (2011). The use of blog in decision making skills course.Procedia Social and Behavioral Sciences, 18, 491-500.

Mansor, A. Z. (2012). Top five creative ideas using web 2.0.Procedia Social and Behavioral Sciences, 59, 429-437.

Mardia, K. V. (1970). Measures of multivariate skewness and kurtosis with applications. Biometrika, 57(3), 519-530

Marín, V. (2013). Innovando en las aulas universitarias a través de los blogs desde una perspectiva inclusiva. RevistaInteracções, 9(23), 88-101.

Marín, V., Muñoz, J. M., y Sampedro, B. E. (2014). Los blogs educativos como herramienta para trabajar la inclusión desde la educación superior. Ensayos, Revista de la Facultad de Educación de Albacete,29(2), 115-127

Marín, V., y Gómez, M ${ }^{a}$ E. (2015). Edublogs in the development of the European higher education area: an initiative in the Primary Education Degree course at the University of Córdoba (Spain). Procedia Social and Behavioral Sciences, 197, 2251-2256.

Marín, V., y Sampedro, B.E. (2016). Los edublogs como herramienta de trabajo en el aula de grado de Educación Primaria. Aula de Encuentro. En prensa.

Mateo, J. (2012). La investigación ex post-facto. In R. Bisquerra (coord.). Metodología de la investigación educativa (pp. 195 - 229). (3a. ed.). Madrid: La Muralla.

Meza, L.G. (2002). Metodología de la investigación educativa: posibilidades de integración. Revista Comunicación, 12(1) (23), 182-194.

Moreno, P. (2015). El sentimiento de autoeficacia en pacientes de oncología infantil: desarrollo de un instrumento de medida. Universidad Complutense de Madrid. Tesis no publicada.

Muthen, B., y Kaplan, D. (1992). A comparison of some methodologies for the factor analysis of non-normal Likert variables: A note on the size of the model. British Journal of Mathematical and Statistical Psychology, 45, 19-30.

Noel, L. (2015). Using blogs to create a constructivist learning environment. Procedia Social and Behavioral Science, 174, 617-621.

Nunually, J.C.(1978). Psychometric Theory. New York: McGraw-Hill.

Osman, G., y Koh, J. H. L. (2013).Understanding management students' reflective practice through blogging. The Internet and Higher Education, 16, 23-31.

Quintanal, F. (2011). Utilización de herramientas Web 2.0 en la Física y Química de Bachillerato. Arbor, 187(3), 153-158 
Rojas, A. C., y Parra, H. (2009). La construcción del conocimiento didáctico matemático al utilizar software educativos. Revista Paradigma, XXX(1), 169-182.

Sabariego, M. (2012). La investigación educativa: Genésis, Evolución y Características. En R. Bisquerra (coord.). Metodología de la investigación educativa (pp.51-88). (3ª ed.). Madrid: La Muralla.

Tapia, M. D., y Escudero, R. (2014). Aprendizaje colaborativo mediante el uso de edublog en la enseñanza universitaria. Valoración de una experiencia. Enseñanza \& Teaching, 32, 53-72.

Zeng, X., y Harris, S. T. (2005).Blogging in an online heath information technology class. Perspective in Heath Information Management, 2(6). 